\title{
Interaction potentials for soft and hard ellipsoids
}

\author{
R. Everaers* and M.R. Ejtehadi ${ }^{\dagger}$ \\ Max-Planck-Institut für Polymerforschung, Postfach 3148, D-55021 Mainz, Germany
}

(May 28, 2018)

\begin{abstract}
Using results from colloid science we derive interaction potentials for computer simulations of mixtures of soft or hard ellipsoids of arbitrary shape and size. Our results are in many respects reminicent of potentials of the Gay-Berne type but have a well-defined microscopic interpretation and no adjustable parameters. Since our potentials require the calculation of similar variables, the modification of existing simulation codes for Gay-Berne potentials is straightforward. The computational performance should remain unaffected.
\end{abstract}

\section{INTRODUCTION}

In molecular simulations [1,2] short-range attractive and repulsive interactions are typically represented using Lennard-Jones (LJ) 6-12 potentials:

$$
U_{\mathrm{LJ}}=4 \epsilon_{\mathrm{LJ}}\left(\left(\frac{\sigma}{r}\right)^{12}-\left(\frac{\sigma}{r}\right)^{6}\right)
$$

where $\sigma / r$ is the dimensionless ratio of the effective particle diameter and the interparticle distance. While the $r^{-6}$ part has a physical origin in dispersion or van der Waals-interactions, the $r^{-12}$ repulsion is chosen by mathematical convenience. For large molecules the evaluation of the interaction potential involves a computationally expensive double summation of Eq. (1) over the respective (atomic) interaction sites

$$
U=\sum_{i \in \text { Body } 1} \sum_{j \in \text { Body } 2} U_{\mathrm{LJ}}\left(r_{i j}\right)
$$

or the evaluation of a double integral

$$
U=\int_{\text {Body } 1} \int_{\text {Body } 2} \rho_{1}(\vec{r}) \rho_{2}\left(\vec{r}^{\prime}\right) U_{\mathrm{LJ}}\left(\left|\vec{r}-\vec{r}^{\prime}\right|\right) d V d V^{\prime}
$$

in the corresponding continuum approximation for bodies with simple geometric shapes and number densities $\rho_{i}(\vec{r})$ of interaction sites. We will refer to interaction energies obtained by (numerically) evaluating Eq. (3) as the "Hamaker" potential.

\footnotetext{
* present address: Max-Planck-Institut für Physik komplexer Systeme, Nöthnitzer Str. 38, 01187 Dresden, Germany

${ }^{\dagger}$ present address: Department of Physics \& Astronomy, University of British Columbia, 6224 Agricultural Road, Vancouver, B.C. V6T 1Z1, Canada
}

As an alternative Gay and Berne [3] (GB) proposed the use of more complicated single-site interaction potentials for rigid molecules. Their approach is based on a heuristic modification of a Gaussian overlap potential. While GB potentials provide a computationally efficient way to introduce anisotropic interactions in numerical studies of liquid crystalline systems [4,5], they have frequently been criticised for their unclear microscopic interpretation [6]. In the present paper, we use results from colloid science [7] to derive approximate interaction potentials for mixtures of ellipsoids of arbitrary size and shape which have a well-defined microscopic interpretation and no adjustable parameters.

The paper is organized as follows: After introducing the Gay-Berne potential in section II, we review in section III the Hamaker theory for two spheres of arbitrary size and develop a relatively simple approximation of the interaction potential which is valid at arbitrary distances. In section IV we generalize this expression to the case of interacting ellipsoids. Section III presents a numerical test of this approximation for the case of pole contacts between aligned ellipsoids. In section VI we suggest computable expressions for the orientational dependence of the interaction potential which are in many respects reminicent of those familiar from GB potentials. A numerical test of the proposed interaction potential for ellipsoidal particles of different shape at arbitrary relative position and orientation is presented in in section VII. We conclude with a brief summary in section VIII.

\section{THE GAY-BERNE POTENTIAL}

A rigid body $i$ is specified by its center position $\vec{r}_{i}$, its orientation (expressed, for example, via a rotation matrix $\mathbf{A}_{i}$ for the transformation from the lab frame to the body frame) and its shape. In the case of ellipsoids, the shape is given by three radii $a_{i}, b_{i}, c_{i}$ which can be used to define a "structure matrix"

$$
\mathbf{S}_{i}=\left(\begin{array}{ccc}
a_{i} & 0 & 0 \\
0 & b_{i} & 0 \\
0 & 0 & c_{i}
\end{array}\right)
$$

in the body frame of the ellipsoid.

The most general form of a Gay-Berne potential for dissimilar biaxial ellipsoids was introduced by Berardi, Fava and Zannoni (BFZ) [8] as a product of three terms:

$$
\begin{aligned}
U\left(\mathbf{A}_{1}, \mathbf{A}_{2}, \vec{r}_{12}\right) & =U_{\mathrm{r}}\left(\mathbf{A}_{1}, \mathbf{A}_{2}, \vec{r}_{12}\right) \\
& \times \eta_{12}\left(\mathbf{A}_{1}, \mathbf{A}_{2}\right) \quad \chi_{12}\left(\mathbf{A}_{1}, \mathbf{A}_{2}, \hat{r}_{12}\right)
\end{aligned}
$$


where $\left(\hat{r}_{12}\right) \vec{r}_{12}$ is the (unit) vector between the center positions: $\vec{r}_{12} \equiv \vec{r}_{2}-\vec{r}_{1}$ and $\hat{r}_{12} \equiv \vec{r}_{12} /\left|\vec{r}_{12}\right|$.

The first term controls the distance dependence of the interaction and has the form of a simple LJ potential $\mathrm{Eq}(1)$

$$
U_{\mathrm{r}}=4 \epsilon_{\mathrm{GB}}\left(\left(\frac{\sigma}{h_{12}+\gamma \sigma}\right)^{12}-\left(\frac{\sigma}{h_{12}+\gamma \sigma}\right)^{6}\right)
$$

where the interparticle distance $r_{12}$ is replaced by the distance $h_{12}$ of closest approach between the two bodies:

$$
h_{12} \equiv \min \left(\left|\vec{r}_{i}-\vec{r}_{j}\right|\right) \forall(i \in \operatorname{Body} 1, j \in \text { Body } 2) .
$$

The position of the potential minimum, $\left(2^{1 / 6}-\gamma\right) \sigma$ is shifted empirically relative to the Lennard-Jones value $2^{1 / 6} \sigma$. Typically $\gamma=1$. The well depth is $\min \left(U_{\mathrm{r}}\right)=$ $\epsilon_{\mathrm{GB}}$.

In general, the calculation of $h_{12}$ is non-trivial. For ellipsoids a suitable scheme was worked out by Perram et al. $[6,9,10]$. These authors also clarified the meaning of the distance

$$
\begin{aligned}
h_{12}^{\mathrm{GB}}\left(\mathbf{A}_{1}, \mathbf{A}_{2}, \vec{r}_{12}\right) & =r_{12}-\sigma_{12}\left(\mathbf{A}_{1}, \mathbf{A}_{2}, \hat{r}_{12}\right) \\
\sigma_{12}\left(\mathbf{A}_{1}, \mathbf{A}_{2}, \hat{r}_{12}\right) & =\left[\begin{array}{lll}
\frac{1}{2} \hat{r}_{12}^{T} & \mathbf{G}_{12}^{-1}\left(\mathbf{A}_{1}, \mathbf{A}_{2}\right) & \hat{r}_{12}
\end{array}\right]^{-1 / 2} \\
\mathbf{G}_{12}\left(\mathbf{A}_{1}, \mathbf{A}_{2}\right) & =\mathbf{A}_{1}^{T} \mathbf{S}_{1}^{2} \mathbf{A}_{1}+\mathbf{A}_{2}^{T} \mathbf{S}_{2}^{2} \mathbf{A}_{2}
\end{aligned}
$$

which is usually employed together with the Gay-Berne potential [11]. Eq. (8) is an approximation which fails, for example, in the case of two spheres with unequal radii $a_{1} \ll a_{2}$ where $\sigma_{12}=\sqrt{2\left(a_{1}^{2}+a_{2}^{2}\right)} \approx \sqrt{2} a_{2} \gg a_{1}+a_{2}$. In this article we always use the correct contact distance $h_{12}$. Fig. $4 \mathrm{a}$ and b provide a comparison of the quality of the various approximation schemes, if $h_{12}$ is replaced by the Gay-Berne approximation $h_{12}^{\mathrm{GB}}$.

The two other terms in Eq. (5) control the interaction strength as a function of the relative orientation and position of the ellipsoids. The second term [12] introduces an empirical exponent $\nu$ :

$$
\begin{aligned}
\eta_{12}\left(\mathbf{A}_{1}, \mathbf{A}_{2}\right) & =\left[\frac{2 s_{1} s_{2}}{\operatorname{det}\left[\mathbf{G}_{12}\left(\mathbf{A}_{1}, \mathbf{A}_{2}\right)\right]}\right]^{\nu / 2} \\
s_{i} & =\left[a_{i} b_{i}+c_{i} c_{i}\right]\left[a_{i} b_{i}\right]^{1 / 2}
\end{aligned}
$$

The third term has the form

$$
\chi_{12}\left(\mathbf{A}_{1}, \mathbf{A}_{2}, \hat{r}_{12}\right)=\left[\begin{array}{ll}
\hat{r}_{12}^{T} & \mathbf{B}_{12}^{-1}\left(\mathbf{A}_{1}, \mathbf{A}_{2}\right) \hat{r}_{12}
\end{array}\right]^{\mu} .
$$

with

$$
\mathbf{B}_{12}\left(\mathbf{A}_{1}, \mathbf{A}_{2}\right)=\mathbf{A}_{1}^{T} \mathbf{E}_{1} \mathbf{A}_{1}+\mathbf{A}_{2}^{T} \mathbf{E}_{2} \mathbf{A}_{2}
$$

and

$$
E_{i}=\left(\begin{array}{ccc}
e_{a i}^{-1 / \mu} & 0 & 0 \\
0 & e_{b i}^{-1 / \mu} & 0 \\
0 & 0 & e_{c i}^{-1 / \mu}
\end{array}\right)
$$

where $e_{a i}, e_{b i}, e_{c i}$ characterize the relative well depth for side-to-side, face-to-face and end-to-end interactions between two ellipsoids of type $i . \mu$ is another empirical exponent.

To summarize, the physical problem of a mixture of colloidal particles of equal composition, but of different sizes and (ellipsoidal) shapes, is defined via Eqs. (1) and (3). It requires the specification of a material constant and of the shapes $a_{i}, b_{i}, c_{i}$ of the involved particles. GayBerne potentials introduce additional adjustable parameters: the shift parameter $\gamma$, the empirical exponents $\nu$ and $\mu$, and three energy parameters per particle type. These parameters are usually adjusted by fitting Eq. (5) to the numerical evaluation of Eq. (2) for small assemblies of suitably arranged Lennard-Jones particles [3] or specific organic molecules [13]. Note, that there are no additional parameters specifying the interactions between ellipsoids of different shape. Rather, Eqs. (6) to (15) provide heuristic "mixing rules" for this case.

In the following we will partially justify the orientation dependent part of the Gay-Berne potential and the implicit mixing rules for particular choices of the adjustable parameters. Note, however, that the product ansatz of Eqs. (5) incorrectly reduces the attractive and repulsive parts of the interaction between extended objects at arbitrary distances to simple power laws with distance independent, shape and orientation dependent prefactors. To overcome this problem we will abandon the strategy initiated by Gay and Berne who sought modifications to the Lennard-Jones potential for point particles. Instead we will try to preserve the case of interacting spheres of finite volume as a proper limit.

\section{HAMAKER THEORY}

Eq. (3) can be solved exactly for two spheres of radius $a_{1} \leq a_{2}$, volume $V_{i}=\frac{4 \pi}{3} a_{i}^{3}$ at a distance $r_{12}=\left(a_{1}+\right.$ $\left.a_{2}\right)+h_{12}$ with $h_{12}>0$. For the attractive part of the interaction Hamaker [14] obtained

$$
\begin{aligned}
U_{A}= & -\frac{A_{12}}{6}\left[\frac{2 a_{1} a_{2}}{r_{12}^{2}-\left(a_{1}+a_{2}\right)^{2}}+\frac{2 a_{1} a_{2}}{r_{12}^{2}-\left(a_{1}-a_{2}\right)^{2}}\right. \\
& \left.+\ln \left(\frac{r_{12}^{2}-\left(a_{1}+a_{2}\right)^{2}}{r_{12}^{2}-\left(a_{1}-a_{2}\right)^{2}}\right)\right]
\end{aligned}
$$

where $A_{12}$ is usually referred to as Hamaker's constant. Using LJ units $A_{12}$ is given by $A_{12}=4 \pi^{2} \epsilon\left(\rho \sigma^{3}\right)^{2}$. Similarly, we found for the repulsive part of the LJ potential:

$$
\begin{aligned}
U_{R}= & \frac{A_{12}}{37800} \frac{\sigma^{6}}{r_{12}}[ \\
& \frac{r_{12}^{2}-7 r_{12}\left(a_{1}+a_{2}\right)+6\left(a_{1}^{2}+7 a_{1} a_{2}+a_{2}^{2}\right)}{\left(r_{12}-a_{1}-a_{2}\right)^{7}} \\
& +\frac{r_{12}^{2}+7 r_{12}\left(a_{1}+a_{2}\right)+6\left(a_{1}^{2}+7 a_{1} a_{2}+a_{2}^{2}\right)}{\left(r_{12}+a_{1}+a_{2}\right)^{7}}
\end{aligned}
$$




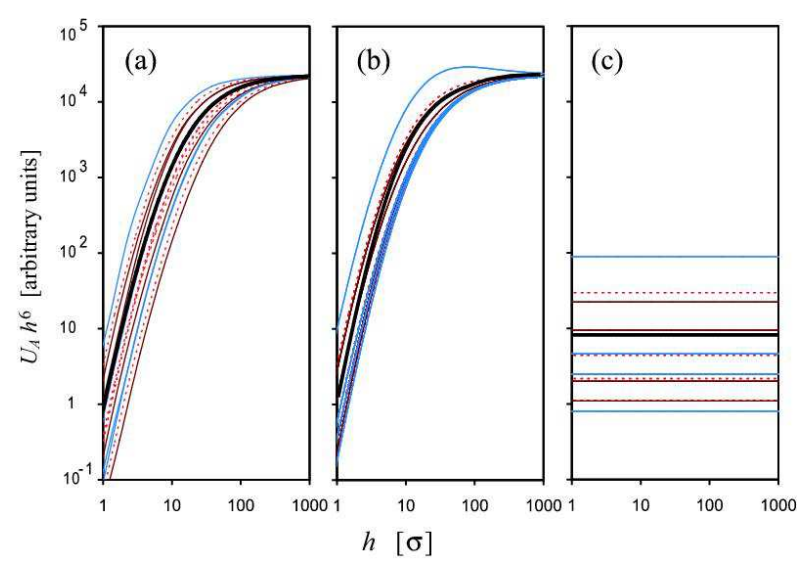

FIG. 1. Attractive part $U_{A}$ of the potential energie multiplied by the inverse asymptotic distance dependence $h^{6}$ as a function of the distance of closest approach $h$ for pole contacts between differently shaped ellipsoids: (a) Hamaker potential, (b) the approximation proposed in the present paper, and (c) a Gay-Berne 6-12 potential adjusted to reproduce the energy minima within the Deryaguin aproximation. The curves converge at large distances, because we have used prolate $((a, b, c)=(1,6,6) \sigma)$, oblate $((a, b, c)=(2,2,9) \sigma)$ and spherical $\left(a=36^{\frac{1}{3}} \sigma\right)$ ellipsoids of identical volume. The plots contain results for sphere-sphere (thick solid line), prolate-prolate (gray lines), oblate-oblate (narrow dark lines), and prolate-oblate (dotted lines) contacts.

$$
\begin{aligned}
& -\frac{r_{12}^{2}+7 r_{12}\left(a_{1}-a_{2}\right)+6\left(a_{1}^{2}-7 a_{1} a_{2}+a_{2}^{2}\right)}{\left(r_{12}+a_{1}-a_{2}\right)^{7}} \\
& \left.-\frac{r_{12}^{2}-7 r_{12}\left(a_{1}-a_{2}\right)+6\left(a_{1}^{2}-7 a_{1} a_{2}+a_{2}^{2}\right)}{\left(r_{12}-a_{1}+a_{2}\right)^{7}}\right]
\end{aligned}
$$

Some insight can be gained by considering three limiting cases: (i) distances which are smaller than the (curvature) radii of the spheres, (ii) a small sphere (i.e. a point particle) at an intermediate distance from a much larger sphere, and (iii) the large distance limit of a 6-12 Lennard-Jones potential with appropriately renormalized prefactor:

$$
\begin{aligned}
& U_{A}= \begin{cases}-\frac{A_{12}}{12} \frac{2 a_{1} a_{2}}{a_{1}+a_{2}} \frac{1}{h_{12}} & \text { for } 0<h_{12} \ll a_{1} \\
-\frac{A_{12}}{6 \pi} V_{1} \frac{1}{h_{12}^{3}} & \text { for } a_{1} \ll h_{12} \ll a_{2} \\
-\frac{A_{12}}{\pi^{2}} V_{1} V_{2} \frac{1}{h_{12}^{6}} & \text { for } a_{1}, a_{2} \ll h_{12}\end{cases} \\
& U_{R}= \begin{cases}\frac{A_{12}}{2520} \frac{2 a_{1} a_{2}}{a_{1}+a_{2}}\left(\frac{\sigma}{h_{12}}\right)^{6} \frac{1}{h_{12}} & \text { for } 0<h_{12} \ll a_{1} \\
\frac{A_{12}}{45 \pi} V_{1}\left(\frac{\sigma}{h_{12}}\right)^{6} \frac{1}{h_{12}^{3}} & \text { for } a_{1} \ll h_{12} \ll a_{2} \\
\frac{A_{12}}{\pi^{2}} V_{1} V_{2}\left(\frac{\sigma}{h_{12}}\right)^{6} \frac{1}{h_{12}^{6}} & \text { for } a_{1}, a_{2} \ll h_{12}\end{cases}
\end{aligned}
$$

Fig. 1a shows a log-log plot of $U_{A} h^{6}$ which illustrates the deviations from the asymptotic power law at small distances. The figure also contains numerical result for the

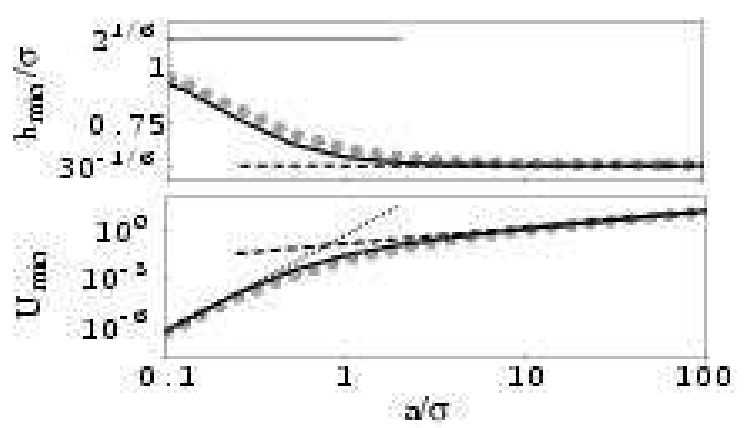

FIG. 2. Sphere size dependence of the position (a) and depth (b) of the energy minimum: Hamaker potential $(\bullet)$ Eqs. (16) and (17), our approximation (-) Eqs. (20) and $(21)$, large/Deryaguin $(---)$ and small/Lennard-Jones $(\cdots)$ sphere limits Eqs. (18) and (19).

attractive part of the Hamaker potential for pole contacts between prolate and oblate ellipsoids. Qualitatively, the curves resemble each other. They converge at large distances, because the particles were chosen to have identical volumes. In contrast, at small distances the interaction strongly depends on the relative orientation and position of the non-spherical particles.

Since Eqs. (16) and (17) are too complicated for an approximate generalization, we have instead developed a suitable combination of the three limiting cases discussed above:

$$
\begin{aligned}
U_{A} \approx & -\frac{A_{12}}{36}\left(1+3 \frac{2 a_{1} a_{2}}{a_{1}+a_{2}} \frac{1}{h_{12}}\right) \\
& \times\left(\frac{a_{1}}{a_{1}+h_{12} / 2}\right)^{3}\left(\frac{a_{2}}{a_{2}+h_{12} / 2}\right)^{3} \\
U_{R} \approx & \frac{A_{12}}{2025}\left(\frac{\sigma}{h}\right)^{6}\left(1+\frac{45}{56} \frac{2 a_{1} a_{2}}{a_{1}+a_{2}} \frac{1}{h_{12}}\right) \\
& \times\left(\frac{a_{1}}{a_{1}+h_{12} / 60^{1 / 3}}\right)^{3}\left(\frac{a_{2}}{a_{2}+h_{12} / 60^{1 / 3}}\right)^{3}
\end{aligned}
$$

Prefactors were chosen in such a way that Eqs. (20) and (21) reproduce the limits Eqs. (18) and (19) of the exact results. As a consequence, the approximation is fairly reliable on all length scales (compare, for example, the thick solid lines in Figs. 1a and b). This is also demonstrated in Fig. 2 where we show the sphere size dependence of the depth and position of the minimum of $U_{R}+U_{A}$. While the short distance expansion becomes reliable for sphere radii $a>5 \sigma$, Eqs. (20) and (21) essentially reproduce the exact results for arbitrary sphere sizes.

As pointed out above, potentials of the Gay-Berne type Eq. (5) cannot describe the complex distance dependence of the interaction. But what about the potential minimum? Within the short distance/large sphere expansion, its depth $U_{\min }$ and position $h_{12, \min }$ are given by: 


$$
\begin{aligned}
U_{\text {min }} & =-\frac{30^{1 / 6}}{14} A_{12} \frac{2 a_{1} a_{2}}{\sigma\left(a_{1}+a_{2}\right)} \\
h_{12, \text { min }} & =30^{-1 / 6} \sigma
\end{aligned}
$$

For the Gay-Berne potential, on the other hand,

$$
\begin{aligned}
U_{\text {min }}^{\mathrm{GB}} & =\epsilon_{\mathrm{GB}} \chi_{12} \eta_{12} \\
\chi_{12} & =\frac{e_{1} e_{2}}{\left(\frac{1}{2}\left(e_{1}^{1 / \mu}+e_{2}^{1 / \mu}\right)\right)^{\mu}} \\
\eta_{12} & =1 \\
h_{12, \text { min }}^{\mathrm{GB}} & =\left(2^{1 / 6}-\gamma\right) \sigma
\end{aligned}
$$

A shift term with

$$
\gamma=2^{1 / 6}-30^{-1 / 6} \approx 0.56
$$

in the distances in Eq. (6) is thus a natural consequence of the insistence on a 6-12 potential. Furthermore, the comparison suggests a relation between the energy scale of the GB potential and the Hamaker constant

$$
\epsilon_{\mathrm{GB}}=A_{12} \frac{30^{1 / 6}}{14}
$$

as well as the choices $\mu=1$ and $e_{i}=a_{i} / \sigma$. Note, that this result represents a first justification for one of the empirical GB mixing rules.

\section{INTERPOLATING BETWEEN THE DERYAGUIN APPROXIMATION AND THE LARGE DISTANCE LIMIT}

In the general case of ellipsoids of arbitrary shape, relative position and orientation, the small and the large distance limit remain (qualitatively) unchanged. However, for strongly non-spherical ellipsoids with $a_{i} \ll c_{i}$ there are new regimes for intermediate distance $a_{i} \ll h \ll c_{i}$. For example, one finds for two thin prolates with semiaxes $(a, a, L), a \ll L$ different power law behavior for parallel, perpendicular, and aligned configurations:

$$
\begin{aligned}
U_{A \|} & =\frac{2 \pi A_{12}}{5} \frac{a^{4} L}{h^{5}} \\
U_{A+} & =\frac{\pi A_{12}}{2} \frac{a^{4}}{h^{4}} \\
U_{A--} & =\frac{A_{12}}{30} \frac{a^{4}}{h^{2} L^{2}}
\end{aligned}
$$

We have found no truly satisfactory approximation that would reproduce all intermediate limiting cases for ellipsoids of arbitrary shape. Nevertheless we have made some progress compared to a simple 6-12 potential. Our somewhat naive strategy for developing an approximation for the general case is (i) to use the Deryaguin approximation in the short distance limit and (ii) to treat ellipsoids as spheres of equivalent volume on length scales which exceed the particle diameters.
In the limit where large particles almost touch, the relevant distances become small compared to the local radii of curvature of the bodies. The short-distance expansion of Eq. (3) in powers of the local curvature radii is known as Deryaguin approximation $[7,15]$. In the most general case [16], each body has two different principal radii of curvature $R_{i}$ and $R_{i}^{\prime}$ at the point of closest approach. Furthermore, the principal axes of the two surfaces can be rotated by an angle $\theta$ relative to each other. The result of the Deryaguin approximation for Lennard-Jones interactions can be written in the form

$$
U_{\mathrm{DW}}\left(h_{12}, \theta\right) \equiv \frac{A_{12}}{12} \chi_{12} \eta_{12}\left(\frac{1}{210}\left(\frac{\sigma}{h_{12}}\right)^{7}-\left(\frac{\sigma}{h_{12}}\right)\right)
$$

where, in analogy to Eq. (5), we tentatively identify the orientation and relative position dependent part with a product of two terms to be specified below:

$\chi_{12} \eta_{12}=$

$$
\frac{2 \sigma^{-1}}{\sqrt{\left(\frac{1}{R_{1}}-\frac{1}{R_{1}^{\prime}}\right)\left(\frac{1}{R_{2}}-\frac{1}{R_{2}^{\prime}}\right) \sin (\theta)^{2}+\left(\frac{1}{R_{1}}+\frac{1}{R_{2}}\right)\left(\frac{1}{R_{1}^{\prime}}+\frac{1}{R_{2}^{\prime}}\right)}}
$$

We note that Eq. (34) defines together with Eq. (28) and (29), a parameter-free 6-12 potential which correctly describes the position and depth of the energy minima for large colloidal particles.

Our second step is the generalization of Eqs. (20) and (21) to the interaction between ellipsoids [17]

$$
\begin{aligned}
& U_{A} \approx-\frac{A_{12}}{36}\left(1+3 \eta_{12} \chi_{12} \frac{1}{h_{12}}\right) \\
& \times\left(\frac{a_{1}}{a_{1}+h_{12} / 2}\right)\left(\frac{b_{1}}{b_{1}+h_{12} / 2}\right)\left(\frac{c_{1}}{c_{1}+h_{12} / 2}\right) \\
& \times\left(\frac{a_{2}}{a_{2}+h_{12} / 2}\right)\left(\frac{b_{2}}{b_{2}+h_{12} / 2}\right)\left(\frac{c_{2}}{c_{2}+h_{12} / 2}\right) \\
& U_{R} \approx \frac{A_{12}}{2025}\left(\frac{\sigma}{h_{12}}\right)^{6}\left(1+\frac{45}{56} \eta_{12} \chi_{12} \frac{1}{h_{12}}\right) \\
& \times\left(\frac{a_{1}}{a_{1}+h_{12} / 60^{1 / 3}}\right)\left(\frac{b_{1}}{b_{1}+h_{12} / 60^{1 / 3}}\right)\left(\frac{c_{1}}{c_{1}+h_{12} / 60^{1 / 3}}\right) \\
& \times\left(\frac{a_{2}}{a_{2}+h_{12} / 60^{1 / 3}}\right)\left(\frac{c_{2}}{b_{2}+h_{12} / 60^{1 / 3}}\right)\left(\frac{c_{2}+h_{12} / 60^{1 / 3}}{c_{2}}\right)
\end{aligned}
$$

This ansatz reproduces at least the intermediate power laws for parallely and perpendicularly oriented thin prolates quite well:

$$
\begin{aligned}
U_{A \|} & =\frac{4 A_{12}}{3} \frac{a^{4} L}{h^{5}} \\
U_{A+} & =\frac{4 A_{12}}{9} \frac{a^{4}}{h^{4}} \\
U_{A--} & =\frac{4 A_{12}}{9} \frac{a^{4}}{h^{4}}
\end{aligned}
$$

In contrast, our ansatz overestimates the potential at intermediate distances for needles aligned along their long 


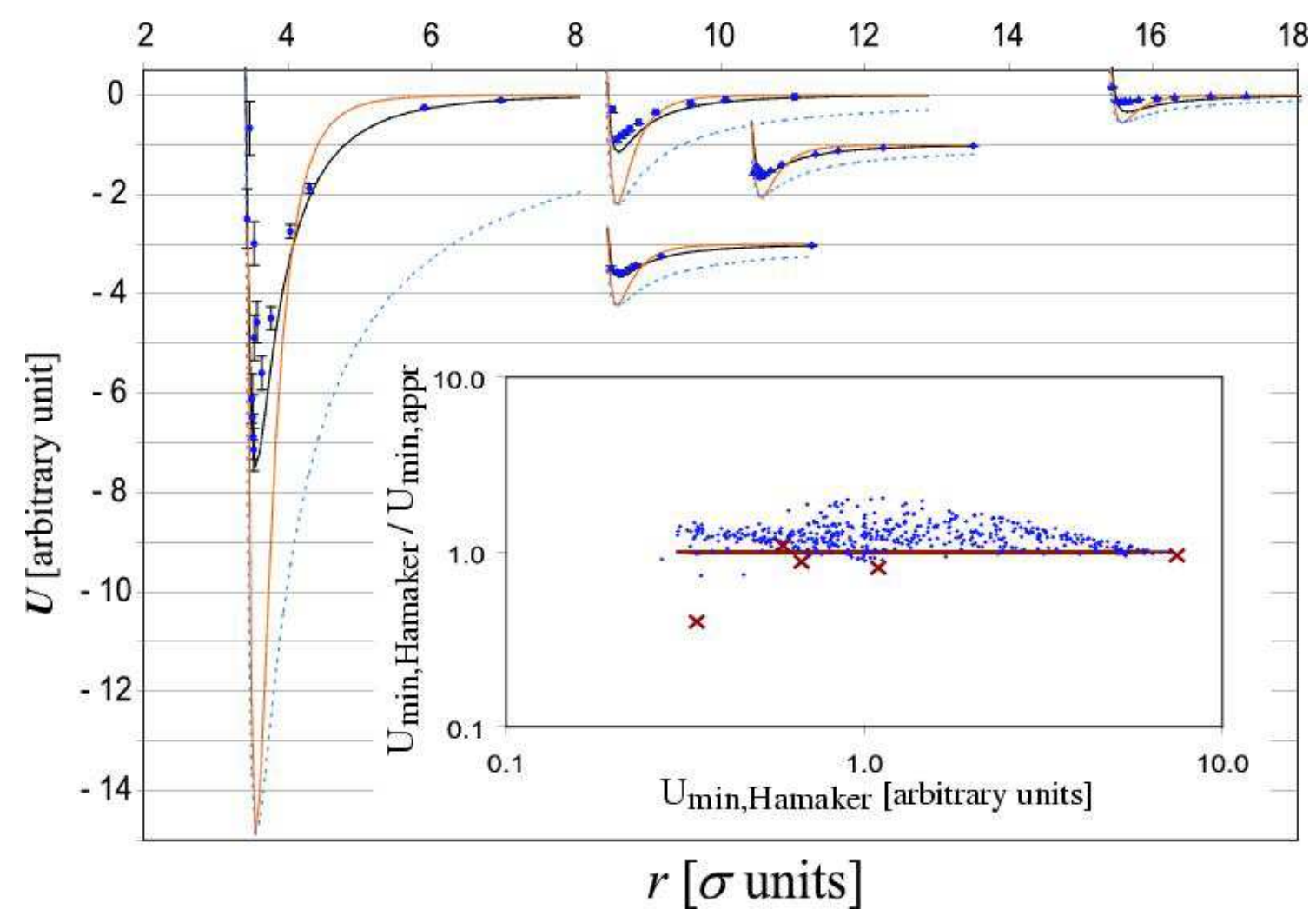

FIG. 3. Distance dependence of the potential energy for all possible pole contacts between an oblate and a prolate ellipsoid with semi-axes $(1,6,6) \sigma$ and $(2,2,9) \sigma$ respectively: $(\bullet)$ Hamaker potential obtained via a Monte Carlo evaluation of the six dimensional integral Eq. (3); (- - ) Deryaguin approximation Eqs. (33) and (40); (...) a GB 6-12 potential Eq. (5) adjusted to reproduce the position and depth of the minima in the Deryaguin approximation Eqs. (28) and (29); (- $)$ our proposal Eqs. (35) and (36). Two data sets were shifted along the $y$-axis for clarity reasons. Note that none of the approximations contains freely adjustable parameters.

The inset shows the ratio of the well depths of the Hamaker potential and of our potential as a function of the absolute well depth. $(\times)$ Results for pole contacts; $(\cdot)$ results for ellipsoids with randomly chosen relative orientations and positions whose distance is varied along the center-to-center line. A comparison of the two data sets allows an evaluation of the quality of our approximations for $\chi_{12}$ and $\eta_{12}$ (see Section VI).

axes. However, the following comparisons will show that the deviations are typically within a factor of two or three over the entire range of distances.

\section{POLE CONTACTS BETWEEN BIAXIAL ELLIPSOIDS}

The evaluation of Eq. (34) is straightforward for pole contacts between aligned ellipsoids with $\mathbf{A}_{1}=\mathbf{A}_{2}$ so that $\theta=0$. For two biaxial ellipsoids which touch at their $c$ poles, the principle curvature radii at the touch point are $R_{i}=\frac{a_{i}^{2}}{c_{i}}$ and $R_{i}^{\prime}=\frac{b_{i}^{2}}{c_{i}}$. In this case Eq. (34) reduces to

$$
\chi_{12} \eta_{12}=\frac{2 \sigma^{-1}}{\sqrt{\left(\frac{c_{1}}{a_{1}^{2}}+\frac{c_{2}}{a_{2}^{2}}\right)\left(\frac{c_{1}}{b_{1}^{2}}+\frac{c_{2}}{b_{2}^{2}}\right)}}
$$

As in the case of interacting spheres, we can check the quality of our approximations by comparing to results obtained by, in the present case numerically, integrating Eq. (3) for pole contacts between oblate and prolate ellipsoids. Fig. 3 shows good agreement for the shape, depth, and position of the minima. In particular, the figure demonstrates that for small molecules Eqs. (35) and (36) provide a significant improvement over the Deryaguin approximation (or a 6-12 potential taylored to reproduce the minima of the Deryaguin approximation). Figs. 1a and $\mathrm{b}$ show that we also reproduce the crossover to the asymptotic behavior at large distances reasonably well, at least relative to the parameter-free Gay-Berne potential (Fig. 1c). 


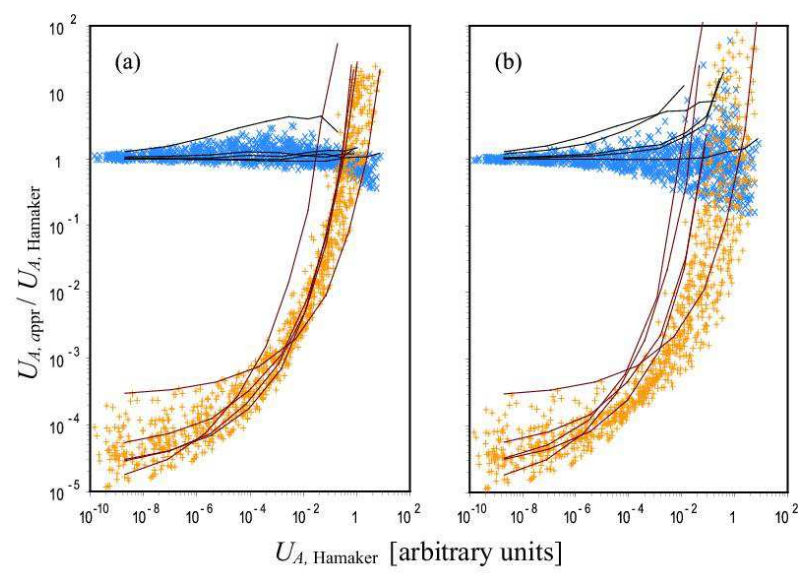

FIG. 4. Deviations of approximate potentials from the true Hamaker potential as a function of the absolute value of the Hamaker potential: (a) using the correct distance of closest approach Eq. (7); (b) using the approximate GB distance function Eq. (8). We show results for contacts between an oblate and a prolate ellipsoid with semi-axes $(a, b, c)=(1,6,6) \sigma$ and $(a, b, c)=(2,2,9) \sigma$ respectively and randomly chosen relative orientations and positions: (dark $\times$ ) our potential, (gray +) a Gay-Berne 6-12 potential adjusted to reproduce the energy minima within the Deryaguin aproximation. The solid lines show the corresponding results for pole contacts (see Fig. 1).

\section{COMPUTABLE EXPRESSIONS FOR ARBITRARY CONTACT GEOMETRIES AND THE RELATION TO THE GAY-BERNE POTENTIAL}

In the next step, we take a closer look at the orientation dependent prefactor in Eq.(33). We are less interested in its exact calculation (i.e. the determination of the contact points, the local curvature radii and the angle between the principle axis on the two surfaces) than in finding expressions which offer a good compromise between computability and correctness. Quite interestingly, our final expressions turn out to be closely related to those used in Gay-Berne potentials.

We begin by providing definitions for $\chi_{12}$ and $\eta_{12}$ which, when multiplied with each other, reproduce Eq. (34) and whose structure resembles Eqs. (13) and (11):

$$
\begin{aligned}
\chi_{12} & \equiv\left(\frac{2 \sigma^{-1}}{\kappa_{1}^{-1}+\kappa_{2}^{-1}}\right) \\
\eta_{12} & \equiv \frac{\kappa_{1}+\kappa_{2}}{\operatorname{det}[\mathbf{Q}(\theta)]^{1 / 2}} \\
\kappa_{i} & =\operatorname{det}\left[\left(\begin{array}{cc}
R_{i} & 0 \\
0 & R_{i}^{\prime}
\end{array}\right)\right]^{1 / 2} \\
\mathbf{Q}(\theta) & \equiv\left(\begin{array}{cc}
R_{1} & 0 \\
0 & R_{1}^{\prime}
\end{array}\right)+\boldsymbol{\Omega}(\theta)^{t}\left(\begin{array}{cc}
R_{2} & 0 \\
0 & R_{2}^{\prime}
\end{array}\right) \boldsymbol{\Omega}(\theta)
\end{aligned}
$$
$\eta_{12}$ can be further simplified for those contacts which dominate in the ordered phases of typical liquid crystals, i.e. contacts between similar poles of identical ellipsoids $\left(a_{1}, b_{1}, c_{1}\right)=\left(a_{2}, b_{2}, c_{2}\right)=(a, b, c)$. 


$$
\begin{aligned}
\chi_{12}\left(\mathbf{A}_{1}, \mathbf{A}_{2}, \hat{r}_{12}\right) & =\frac{4 \sigma^{-1} \operatorname{det}[\mathbf{S}]}{\sigma_{12}^{2}\left(\mathbf{A}_{1}, \mathbf{A}_{2}, \hat{r}_{12}\right)} \\
\eta_{12}\left(\mathbf{A}_{1}, \mathbf{A}_{2}\right) & =\frac{2^{3 / 2} \operatorname{det}[\mathbf{S}]}{\left(\operatorname{det}\left[\mathbf{G}_{12}\right]\right)^{1 / 2}}
\end{aligned}
$$

All in all, we are lead to expressions which are in remarkable agreement with those proposed by BFZ. In the case of $\chi_{12}$, we employ the same functional form together with particular choices for the four adjustable parameters $\mu$, $e_{a i}, e_{b i}, e_{c i}$. In the case of $\eta_{12}$, our Eqs. (50) and (51) respectively Eq. (53) resemble the corresponding Eqs. (11) and (10) so strongly, that it seems clear that our consideration eliminate with $\nu \equiv 1$ the last remaining free parameter of the GB potential.

We note that our proposed modifications leave with $\chi_{12}$ and $\eta_{12}$ the most CPU time intensive part of the GB potential essentially unchanged. The small number of additional scalar operations necessary for the evaluation of Eqs. (35) and (36) hardly affects the performance of simulation codes.

\section{NUMERICAL TEST OF THE APPROXIMATIONS FOR ARBITRARY RELATIVE POSITION AND ORIENTATION OF THE ELLIPSOIDS}

The most important question is, of course, how reliable the proposed approximations are. Similarly to Eqs. (35) and (36), the combination of Eqs. (13), (45), (47), (50) and (51) can only be considered as an educated guess for Eq. (34). The fact that we reproduce the results of the Deryaguin approximation for pole contacts inspires some confidence, but otherwise we have made substancial and uncontrolled approximations which need to be checked against the numerical evaluation of Eq. (3) for various relative positions and orientations. We represent the results by plotting the ratio of the approximative and the exact energy as a function of the exact energy. In this manner, results from a high-dimensional parameter space are (i) projected onto a single axis and (ii) sorted by importance. The inset in Fig. 3 shows a comparison of the depths of the energy wells close to contact for fixed random orientations, while Fig. 4a deals with the attractive part of the interaction at arbitrary distances. Both figures also contain results for the pole contacts discussed before to allow for an independent evaluation of the quality of the approximations for the distance and for the orientational part of the interaction potential.

When judged against the corresponding results for the Gay-Berne potential, the agreement between our proposal and the numerical evaluation of Eq. (3) is excellent. In absolute terms, the deviations do not exceed a factor of two to three in either direction. Quite interestingly, our approximations for $\chi_{12}$ and $\eta_{12}$ do not seem to be the source of large additional errors. Fig. $4 \mathrm{~b}$ shows that the agreement is significantly reduced, if the approximate
GB distance function Eq. (8) is used instead of the true distance of closest approach Eq. (7).

\section{SUMMARY}

We have presented an approximative interaction potential for soft ellipsoidal particles. Our potential uses (almost) the same variables as the Berardi, Fava and Zannoni [8] form of the Gay-Berne [3] potential for biaxial ellipsoids, agrees significantly better with the numerically evaluated exact interaction potential, has no unphysical limits, and avoids the introduction of empirical adjustable parameters. The main modifications we propose are

1. to abandon the unphysical factorization of the orientation and distance dependent parts of the potential (Eq. 5) as well as the Lennard-Jones like form of the distance dependence itself (Eq. 6) and to replace them by Eqs. (35) and (36).

2. to use the Gaussian curvatures at the ellipsoid poles Eqs. (45) - (47) in order to characterize the relative well depth for side-to-side, face-to-face and end-toend interactions through the orientation and relative position dependent factor $\chi_{12}\left(\mathbf{A}_{1}, \mathbf{A}_{2}, \hat{r}_{12}\right)$ defined in Eqs. (13) and (14).

3. to replace the definition Eqs. (10) - (12) of the purely orientiation dependent factor $\eta_{12}\left(\mathbf{A}_{1}, \mathbf{A}_{2}\right)$ by Eqs. (49) - (51).

4. to use the (effective) Hamaker constant $A_{12}$ [7] to set the energy scale.

Our results for the attractive part of the soft potential are directly applicable to hard ellipsoids with van-der-Waals interactions. Furthermore, the proposed potential comprises the interaction of point particles with ellipsoids as a well defined limit. This may be of interest for studies of wetting or polymer adsorption in colloidal dispersions which so far assume either a flat or a cylindrical geometry [18].

\section{ACKNOWLEDGEMENTS}

We gratefully acknowledge extended discussions with L. Delle Site, M. Allen and F. Müller-Plathe and benefitted from a copy of a Gay-Berne simulation code provided by M. Allen. The authors thank the DFG for the financial support of this work within an Emmy-Noether grant. 
[1] M.P. Allen and D.J. Tildesley, Computer simulation of Liquids. (Oxford University Press, Oxford, England, 1989).

[2] D. Frenkel and B. Smit, Understanding Molecular Simulations 2nd ed. (Academic, New York, 2002).

[3] J. G. Gay and B. J. Berne, J. Chem. Phys. 74, 3316 (1981).

[4] M. P. Allen in Advances in the Computer Simulation of Liquid Crystals, edited by P. Pasini and C. Zannoni (Kluwer, Dordrecht, 1999).

[5] E. de Miguel and C. Vega, J. Chem. Phys. 117, 6313 (2002).

[6] J. W. Perram, J. Rasmussen, E. Praestgaard , and J. L. Lebowitz, Phys. Rev. E 54, 6565 (1996)

[7] R. J. Hunter, Foundations of Colloid Science 2nd ed. (Oxford University press, Oxford, England, 2001).

[8] R. Berardi, C. Fava and C. Zannoni, Chem. Phys. Lett. 236, 462 (1995).

[9] J. W. Perram and M.'S. Wertheim, J. Comput. Phys. 58, 409 (1985)

[10] J.'W. Perram, M.'S. Wertheim, J. L. Lebowitz, and G. O. Williams, Chem. Phys. Lett. 105, 277 (1984)

[11] D. J. Cleaver et al, Phys. Rev. E 54, 559 (1996)

[12] There is a slight problem with the BFZ suggestion for $\eta_{12}$, because the definition of the numerator leads to different energies for two physically indistinguishable situations: (i) rotation of an ellipsoid by 90 degrees around its $b$-axis and (ii) exchange of the values of $a_{i}$ and $c_{i}$.

[13] R. Berardi, C. Fava and C. Zannoni, Chem. Phys. Lett. 297, 8 (1998).

[14] H. C. Hamaker, Physics 4, 1058 (1937)

[15] B. V. Deryaguin, Kolloid Z. 69, 155 (1934)

[16] L. R. White, J. Coll. Interf. Sci. 95, 286 (1983).

[17] The functional form can at least partially be justified by the observation that the integrations in Eq. (3) are of a type which produces simple results in two limiting cases:

$$
\begin{aligned}
& \int_{-d / 2}^{d / 2}(r+x)^{-n} d x \\
& \sim \begin{cases}h^{-(n-1)} & \text { for } h=r-d / 2 \ll d \\
d h^{-n} & \text { for } h \approx r \gg d\end{cases} \\
& \sim h^{-(n-1)} \frac{d}{d+h}
\end{aligned}
$$

(i) For separations $h$ which are much smaller than the particle extension the upper limit of the integration can be extended to infinity, reducing the exponents in the power laws describing the interaction by one. (ii) For large separations, the functional form of the interaction is not affected by the integration which only produces a prefactor of the order of the respective particle diameter.

[18] A. Milchev and K. Binder, J. Chem. Phys. 117, 6852 (2002). 